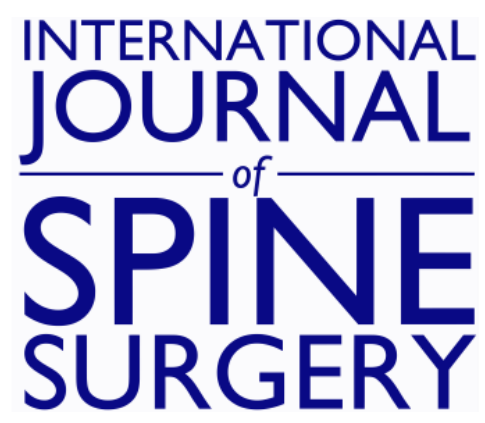

\title{
Risks of Intradiscal Orthobiologic Injections: A Review of the Literature and Case Series Presentation
}

Mairin A. Jerome, Christopher Lutz and Gregory E. Lutz

Int J Spine Surg 2021, 15 (s1) 26-39

doi: https://doi.org/10.14444/8053

http://ijssurgery.com/content/15/s1/26

This information is current as of April 25, 2023.

Email Alerts Receive free email-alerts when new articles cite this article. Sign up at:

http://ijssurgery.com/alerts

The International Journal of Spine Surgery

2397 Waterbury Circle, Suite 1,

Aurora, IL 60504, Phone: +1-630-375-1432 


\title{
Risks of Intradiscal Orthobiologic Injections: A Review of the Literature and Case Series Presentation
}

\author{
MAIRIN A. JEROME, MD ${ }^{1}$ CHRISTOPHER LUTZ, MD, ${ }^{1,2,3}$ GREGORY E. LUTZ, MD ${ }^{1,2,3}$ \\ ${ }^{I}$ Regenerative SportsCare Institute, New York, New York ${ }^{2}$ Department of Physiatry, Hospital for Special Surgery, New York, New York ${ }^{3}$ Department of \\ Rehabilitation Medicine, Weill Cornell Medical College, New York, New York
}

\begin{abstract}
Background: Intervertebral disc disease (IDD) is responsible for a large portion of back pain with historically suboptimal treatments for long-term improvement. IDD pathogenesis is thought to arise at a cellular and biochemical level, making biologically based injections an area of clinical interest. Although human studies have shown promise, emerging data suggest there may be risks inherent to such injections that were previously unrecognized. The aim of this review is to summarize the known risks to date and provide mitigation steps to reduce potential complications in the future. In addition, we present a small case series of serious adverse events (SAEs) from our clinical practice.

Methods: A literature review was performed to identify human intradiscal autologous biologic injection studies to date, including mesenchymal signaling cells (MSCs) and platelet-rich plasma (PRP) preparations, which were reviewed for complications. Cases of complication following intradiscal orthobiologic injection were identified from a single outpatient center and reviewed.

Results: Publications of MSC-based intradiscal injection documented 136 total patients treated with two SAEs reported, one infection and one progressive disc herniation. Publications of PRP intradiscal injection included 194 patients with one SAE reported. We also review three cases of previously unpublished SAEs, including one case of confirmed infection with Cutibacterium acnes ( $C$ acnes) and two presumed cases of discitis without pathogen confirmation. Bone marrow concentrate was the injectate in all three cases.

Conclusions: Although biologic intradiscal injection shows promise for the treatment of discogenic back pain, there are inherent risks to be considered and mitigated. We currently recommend a leukocyte-rich PRP and a two-needle delivery technique coupled with intradiscal gentamicin to mitigate the risk of postinjection spondylodiscitis. Further research is needed using large registries to not only track clinical outcomes but also complication rates.
\end{abstract}

Complications

Keywords: intradiscal biologics, platelet-rich plasma, bone marrow concentrate, complications, percutaneous injection

\section{INTRODUCTION}

Low back pain is a leading cause of disability with a large associated socioeconomic burden from direct treatment-related healthcare expenditure and indirect cost from decreased productivity. ${ }^{1,2}$ Disruption of intervertebral discs (IVDs) accounts for approximately $22 \%$ of back pain cases. ${ }^{3}$ The IVD is a unique structure with a high water content and particular glycosaminoglycan-to-collagen ratio, allowing for tolerance of mechanical stress from multidirectional force vectors. ${ }^{4}$ However, the anatomic and molecular structure makes the IVD particularly susceptible to injury via supraphysiologic mechanical load. ${ }^{5}$ In addition, the disc microenvironment is hypoxic, acidic, and poorly vascularized, and thereby relies on diffusion for nutrient absorption and the elimination of waste. ${ }^{6}$
For this reason, IVDs can contribute to chronic pain via multiple pathways due to diminished inherent healing ability once injured or degenerated. $^{7}$

Many treatments for disc-mediated back pain have been tried, including conservative management with physical therapy, epidural steroid injections, a variety of intradiscal injections, denervation of the annulus, and surgical intervention. ${ }^{7}$ Minimally invasive treatments have historically shown limited success, and there is debate whether surgical intervention for discogenic axial low back pain improves outcome over nonoperative treatment. ${ }^{8}$ More recently, animal models have shown promise using biologic materials, including a variety of individual growth factors, platelet-rich plasma (PRP), and mesenchymal signaling cell (MSC) preparations, to improve water content, disc height, 
and cell proliferation in degenerative IVDs. ${ }^{9,10}$ In humans, several studies have demonstrated functional improvements and global decreases in pain following intradiscal injection of MSCs and/or PRP. ${ }^{11-16}$ Indeed, 2019 American Society of Interventional Pain Physicians practice guidelines were updated to demonstrate level 3 evidence for intradiscal PRP and MSC injections. ${ }^{17}$ This was further supported by a consensus statement published by the American Society of Interventional Pain Physicians in 2020 regarding the evidence-based use of bone marrow concentrate (BMC) for musculoskeletal disorders. ${ }^{18}$

Although early clinical reports are promising, there is an inherently higher risk of injection within the intradiscal space due to its harsh environment compared with other musculoskeletal tissues for which biologic injectates are indicated, such as tendons, ligaments, or joints. Although the procedures have a high overall safety profile, complications have been reported. The purpose of this review is to catalog published complications following intradiscal biologic injection, present a case series of previously unpublished cases, and review safety considerations as well as mitigation strategies to optimize patient safety when performing such procedures.

\section{METHODS}

A literature review was performed using search terms of ((intervertebral disc) OR (intervertebral disk)) AND ((stem cell) OR (bone marrow) OR (platelet rich plasma) OR (regenerative medicine)) AND (injection) with a human species filter to identify relevant studies in two unique databases. The databases queried included PubMed and Cochrane Reviews. The results were compiled and articles were reviewed. Those studies involving any form of autologous mesenchymal stromal cells injected percutaneously into lumbar IVDs in humans were included. Studies using biologics to augment surgery, animal studies, or unrelated papers were excluded. Primary research articles were identified and cross-referenced with review articles to maximize the identification of studies. Case reports, case series, and randomized controlled trials were included and analyzed for the number of complications reported. Publications documenting the same cohort over time were included once as the final publication or were otherwise noted.

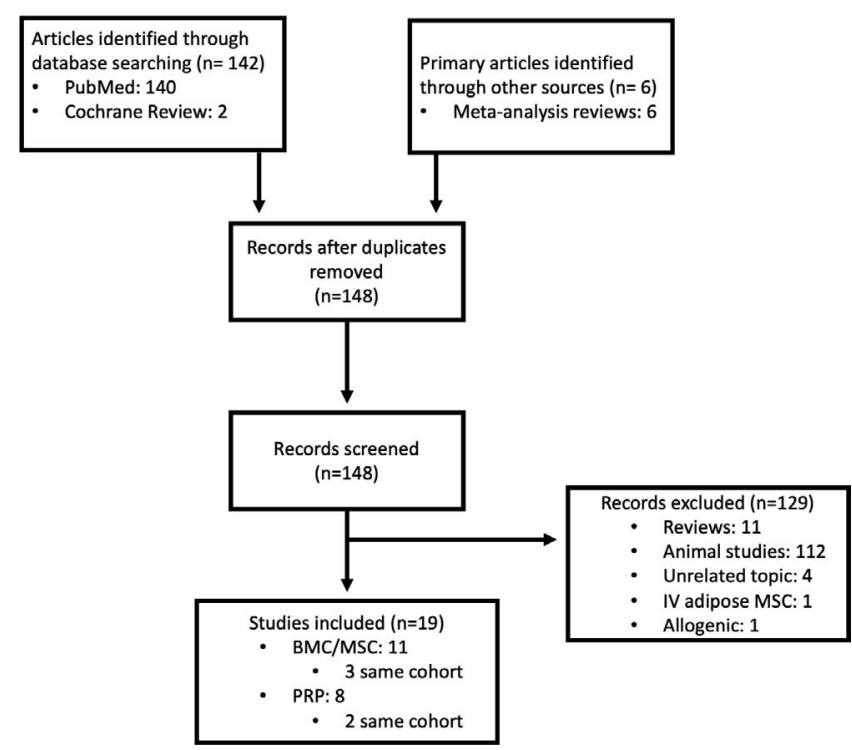

Figure 1. Flowchart outlining literature review and identification of primary articles.

In addition to a review of the literature for identification of previously reported complications following intradiscal orthobiologic injection, we present three cases known to a single, private interventional sports and spine practice. These cases were reviewed for patient characteristics, type of complication, injectate, laboratory values, treatments, and outcomes.

\section{RESULTS}

The PubMed and Cochrane Review search yielded 142 unique publications, respectively. Review papers from the search identified an additional 6 studies. No duplicates were identified. One hundred twenty-nine abstracts were reviewed and excluded, leaving 19 studies meeting inclusion criteria. Of these, 11 were original studies evaluating autologous bone marrow-based injections ( 3 of the same cohort), ${ }^{12,14-16,19-25}$ and 8 were original studies evaluating autologous platelet-based injections ( 2 of the same cohort) $)^{11,26-33}$ (Figure 1). Eleven articles were review papers. ${ }^{34-44}$ Two patients were reported to have major complications. Subach et $\mathrm{al}^{24}$ published a case report of a patient treated at an outside institution with an autologous adipose graft and $\mathrm{BMC}$ with resultant cauda equina syndrome secondary to spondylodiscitis and epidural abscess. In a retrospective cohort study by Centeno et al, ${ }^{16}$ of 33 patients undergoing intradiscal injection of hypoxic cultured bone marrow derived mesenchymal stem cells (BM-MSCs), 1 patient had a 
progressive disc herniation at 3 months. Otherwise, a small number of patients had self-limited postprocedural pain. It is unclear whether the disc herniation was related to the injection because it occurred in a remote fashion. Infection does appear to be related to the procedure, thereby suggesting a complication rate of $0.74 \%$ for intradiscal MSC injection within the published literature. Nine publications document intradiscal injection of PRP with a total of 194 patients treated. One major complication was published as a case report, suggesting a complication rate of intradiscal PRP of $0.52 \%$ (Table 1 ). In addition to the previously published cases, here we present three additional cases of adverse events following biologic intradiscal injection.

\section{Case 1}

An otherwise healthy 55-year-old male nonsmoker was initially seen in consultation for axial low back pain without radiculopathy in June 2019 by author GL at a private outpatient spine and musculoskeletal practice. He failed conservative treatment with physical therapy, oral corticosteroids, and cyclobenzaprine. Previous injections included Regenokine administration to his facet joints at a different institution with partial relief. His physical exam was notable for pain in extreme ranges of flexion and extension. He had no dural tension signs and an intact neurological examination. Magnetic resonance imaging (MRI) showed a mild disc bulge at L4-5 with moderate degeneration at L5-S1 and facet arthrosis, left greater than right, at the lower lumbar segments. There was no significant canal or nerve root compromise. Due to ongoing discogenic pain that was significantly interfering with the patient's quality of life and ability to be active, he elected to undergo L4-5 and L5-S1 intradiscal injections with BMC as well as $\mathrm{BMC}$ injections to the left lumbar facet joints. The procedure was performed on August 8, 2019, using a Marrow Cellutions Bone Aspiration System (Ranfac Corporation, Avon, MA) with a unilateral draw from the posterior superior iliac spine. A total of $60 \mathrm{~mL}$ was aspirated and then further concentrated to $8 \mathrm{~mL}$ using a bone marrow concentration device (EmCyte Corporation, Fort Myers, FL). A sterile preparation and draping was performed. Following intravenous (IV) administration of cefazolin, intradiscal injections were performed using a double-needle technique with an 18-gauge introduc- er needle and a 22-gauge needle threaded to inject the disc. Omnipaque contrast dye, $0.5 \mathrm{~mL}$, combined with $500 \mathrm{mcg}$ of gentamicin was used for confirmation, followed by a $2-\mathrm{mL}$ injection of BMC at each level. The left L4-5 and L5-S1 facet joints were injected under fluoroscopy with $1 \mathrm{~mL}$ of BMC at each level. There was moderate to severe concordant pain with injection of the L5-S1 disc with a grade 3 annular fissure noted (Figure 2A-D).

The patient had some moderate postprocedural pain controlled with $50 \mathrm{mg}$ of tramadol and $10 \mathrm{mg}$ of cyclobenzaprine. On day five postinjection, he developed constipation. He subsequently traveled to Spain with continued increasing crescendo pain with peak severity resulting in presentation and admission to a hospital on day 19 postinjection. Despite not having any systemic symptoms of fever, chills, or sweats, he was admitted for severe pain and found to have a C-reactive protein (CRP) level of $115.5 \mathrm{mg} / \mathrm{L}$, an erythrocyte sedimentation rate (ESR) of $35(\mathrm{~mm} / \mathrm{hr})$, and a white blood cell count of $8.5910^{9} / \mathrm{L}$. An MRI with and without contrast was notable for endplate changes consistent with L5-S1 spondylodiscitis and enhancement of the L4L5 facet joints with extension into the right L4-L5 paravertebral space and the right psoas. No biopsy or cultures were performed, and the patient was treated with 6 weeks of IV cloxacillin followed by oral rifampicin and moxifloxacin for an additional 6 weeks. Three months after his initial admission, he returned for follow-up with no further active signs of infection. His MRI showed interval resolution of the spondylodiscitis (Figure 2E-H), and he was increasing his activity with physical therapy and no longer required opiate pain medication.

\section{Case 2}

A 35-year-old male nonsmoker was seen by author GL on May 6, 2020, for consultation and management of presumed spondylodiscitis following intradiscal injection with BMC performed at a different institution on March 13, 2020. The patient had a medical history significant for traumatic injury to his low back with subsequent L4-L5 laminectomy and microdiscectomy 5 years before presentation. This surgery was revised 1 year later and complicated by cerebral spinal fluid leak. He continued to have refractory pain and disc extrusions at L3-4, L4-5, and L5-S1 with right-sided radiculitis. He underwent intradiscal BMC injections at L3-4 and L4-5 without immediate compli- 


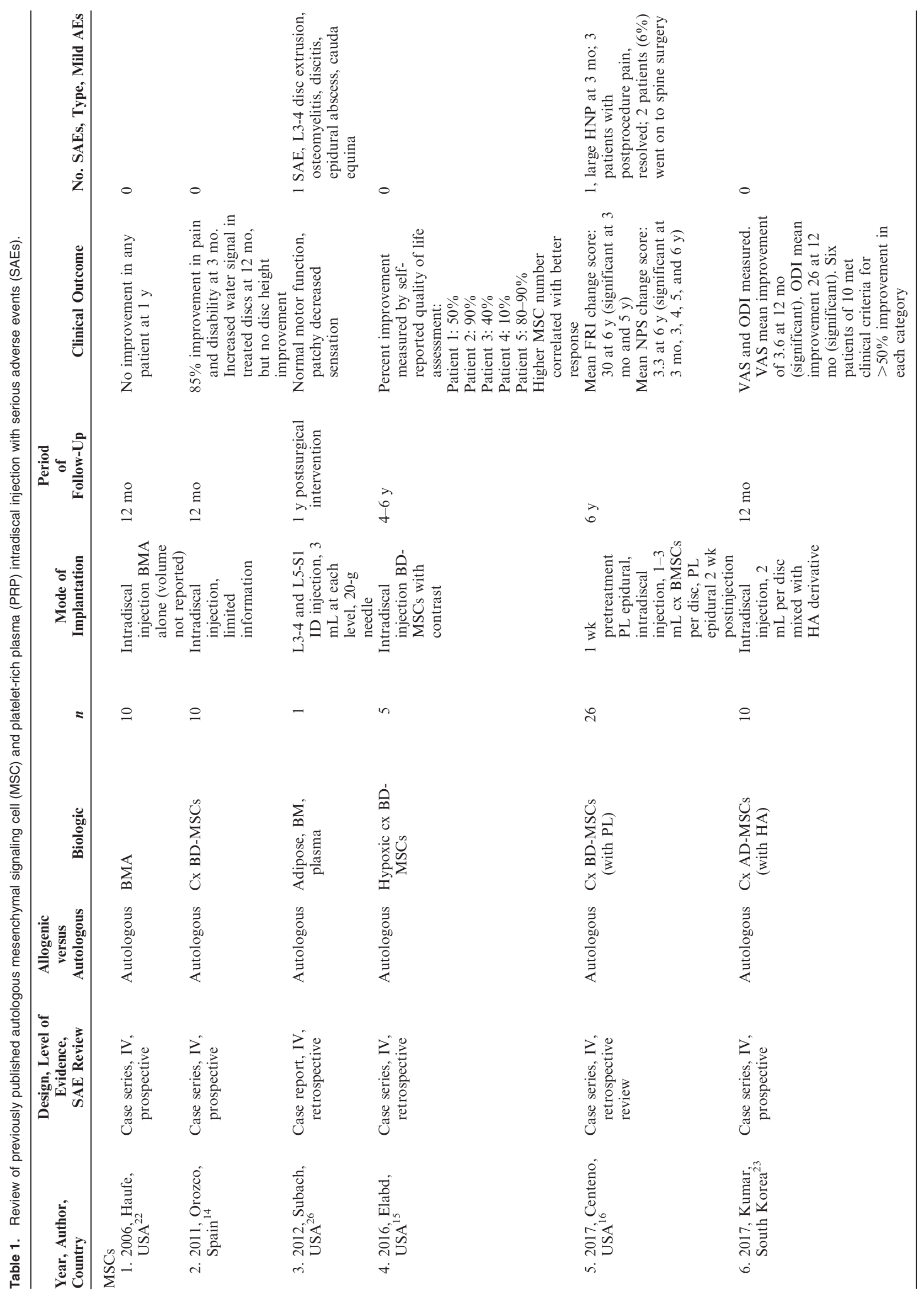




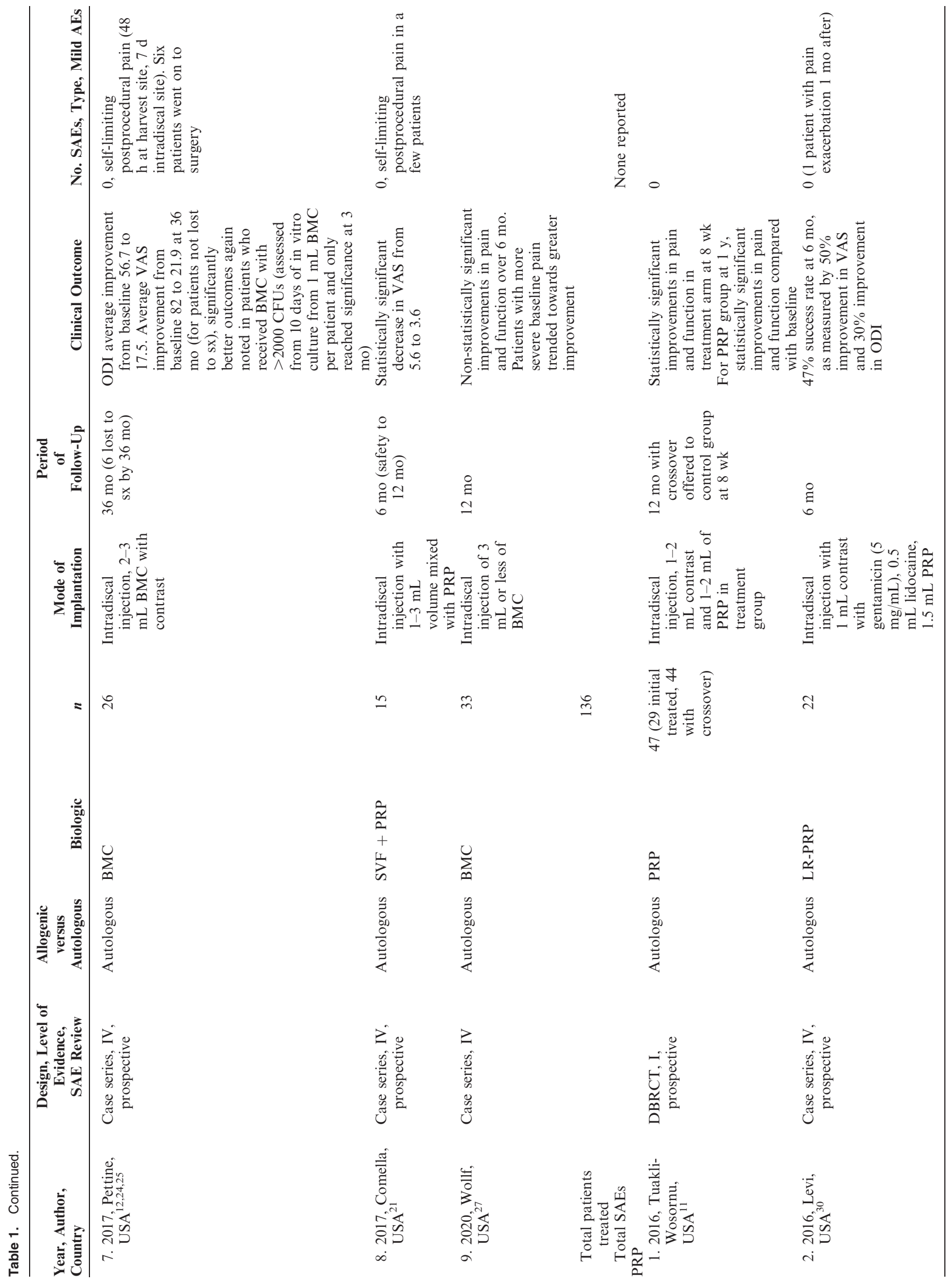




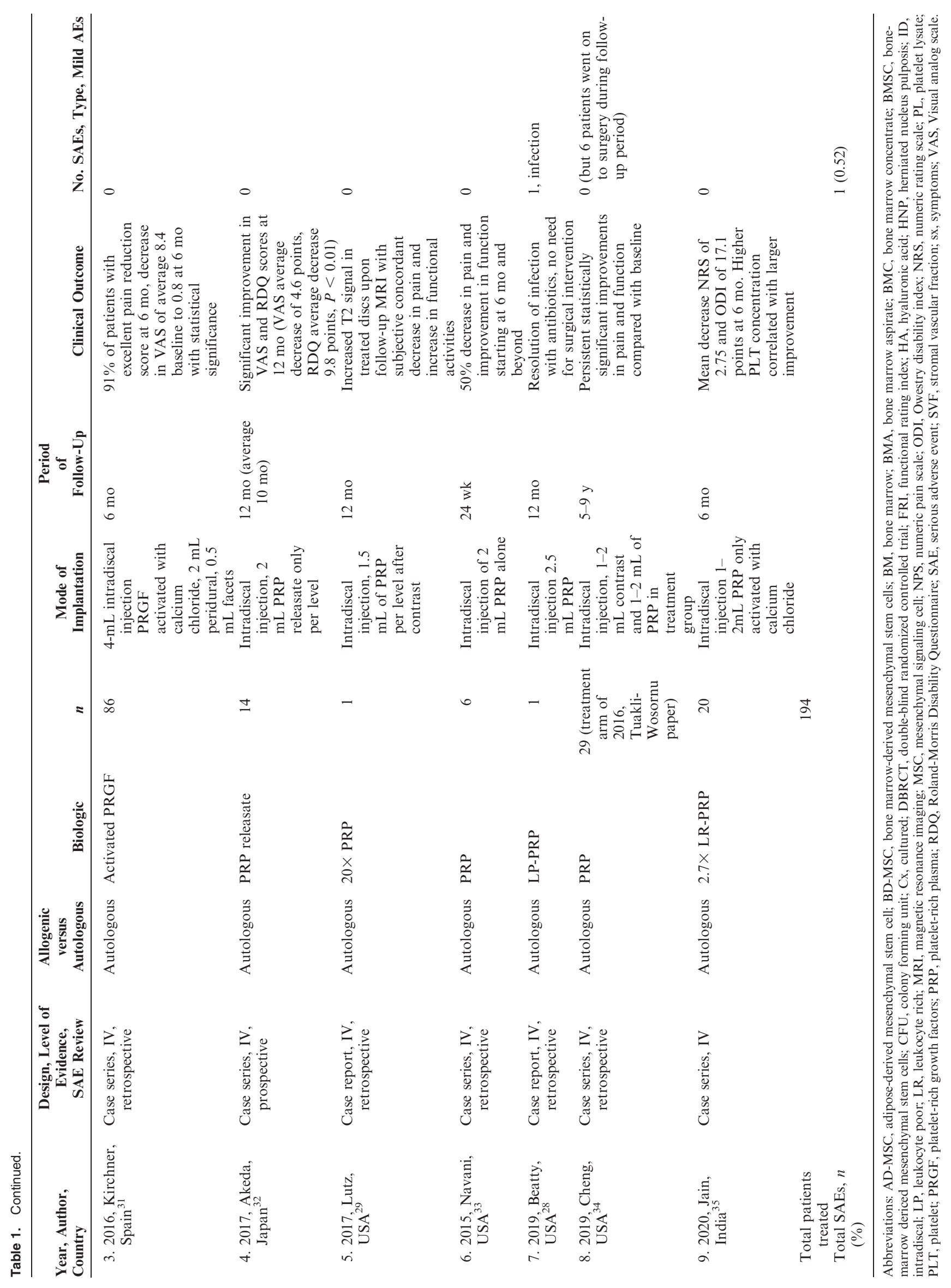




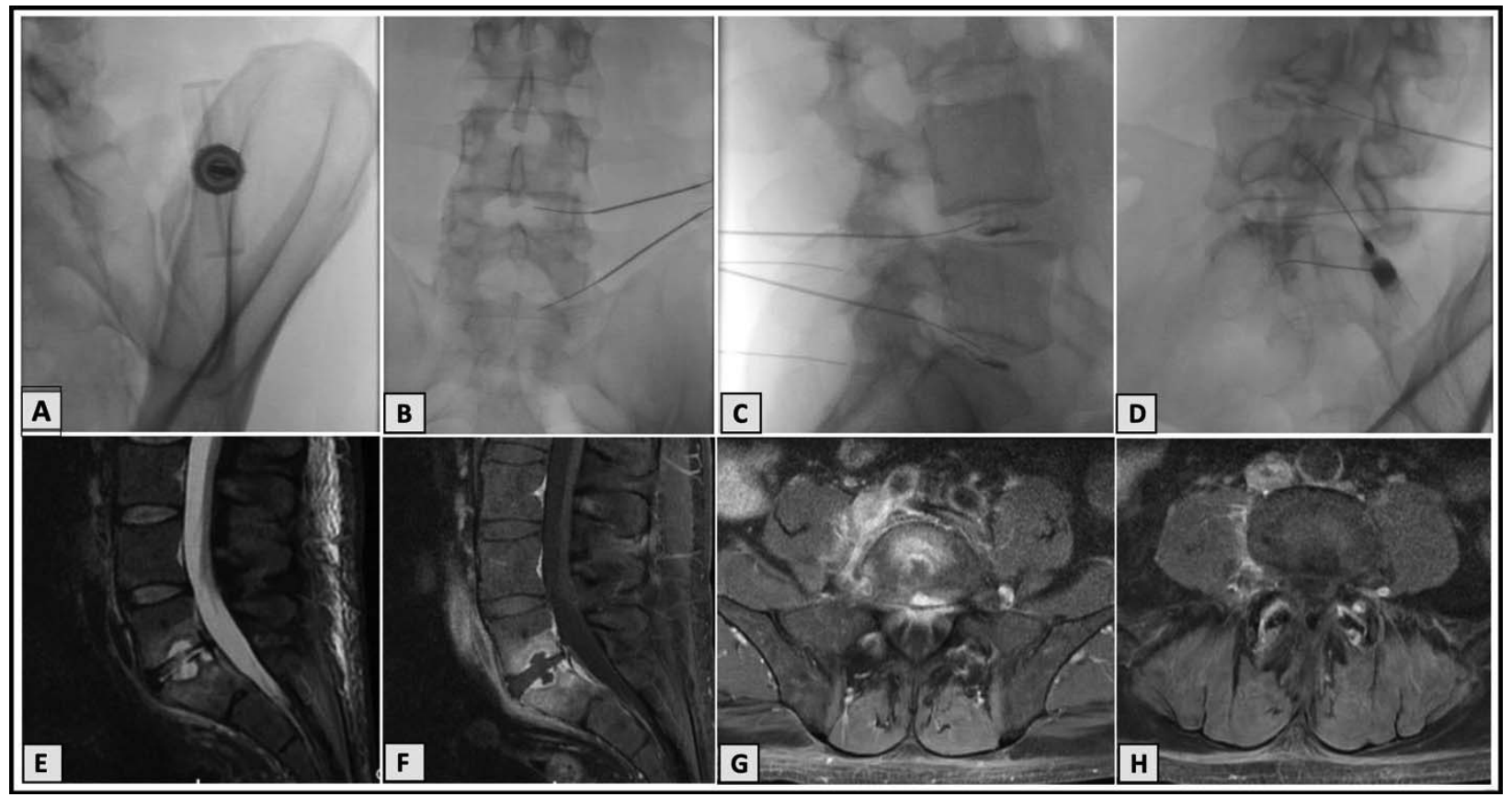

Figure 2. Case 1. Procedural imaging and magnetic resonance imaging (MRI) 8 weeks postprocedure. (A) Fluoroscopy demonstrating bone marrow aspiration. (B) Anteroposterior view fluoroscopy demonstrating intradiscal procedure. (C) Lateral view fluoroscopy demonstrating intradiscal injection with discograms. (D) Contralateral oblique fluoroscopy demonstrating bone marrow concentrate (BMC) intra-articular facet injections at L3-4, L4-5, and L5-S1. (E) T2-weighted lumbar MRI with sagittal view demonstrating endplate changes at L5-S1. (F) T1-weighted fat-saturated lumbar MRI with contrast in sagittal view also demonstrating endplate changes with Schmorl's nodes. (G) T1-weighted fat-saturated postcontrast lumbar MRI axial view demonstrating end plate changes at S1. (H) T1-weighted fatsaturated postcontrast lumbar MRI axial view demonstrating enhancement of the L4-L5 facet joints with extension into the right L4-L5 paravertebral space.

cations. He was given $1 \mathrm{~g}$ of IV cefazolin before the procedure. Autologous BMC (3 mL) harvested from a unilateral posterior superior iliac spine were injected into the L3-L4 and L4-L5 discs. The patient had usual postprocedural pain for 2 days followed by a period of significant pain improvement. However, on day seven, he developed sudden-onset severe low back pain, fever, and constipation. He presented to the hospital with admission and extensive infection workup for fever of unknown origin. MRI of the lumbar spine was concerning for L3-L4 discitis with epidural abscess (Figure 3). His white blood cell count on admission was $6.310^{9 /} \mathrm{L}$, CRP was $4.9 \mathrm{mg} / \mathrm{L}$, and ESR was $49(\mathrm{~mm} / \mathrm{hr})$. Blood cultures were negative. He underwent a computed tomography (CT)-guided aspiration of the spinal fluid with no growth at $>14$ days. No antibiotics were administered before the fluid collection aspiration. The remainder of the workup was negative. Despite negative bacterial culture, he was treated for presumed spondylodiscitis with 6 weeks of IV cefepime and vancomycin. Due to persistent pain following treatment with broad spectrum antibiotics, the treating infectious disease physician administered high-dose dexamethasone with marked symptomatic improvement within 24 hours.

Although significantly improved since his hospitalization, the patient had continued pain 5 months following his initial injections. He underwent a surgical consultation, but was not offered surgery at that time. To date, no organism or confirmation of infection has been established, and his bloodwork has normalized.

\section{Case 3}

A 34-year-old male nonsmoker presented for evaluation of low back pain with radiculopathy secondary to a disc bulge at L4-5 and disc extrusion at L5-S1. His only medical history was degenerative lumbar disc disease. After failing conservative management, he underwent L4-5 and L5-S1 intradiscal injections with a leukocyte-poor PRP on two occasions in tandem with a structured rehabilitation program. He had minimal to no improvement clinically or on imaging following these injections. One year later, due to refractory low back pain, he was offered and underwent intradiscal BMC as an orthobiologic treatment option on February 13, 2018, with author CL. He underwent a unilateral posterior superior iliac spine bone marrow aspira- 


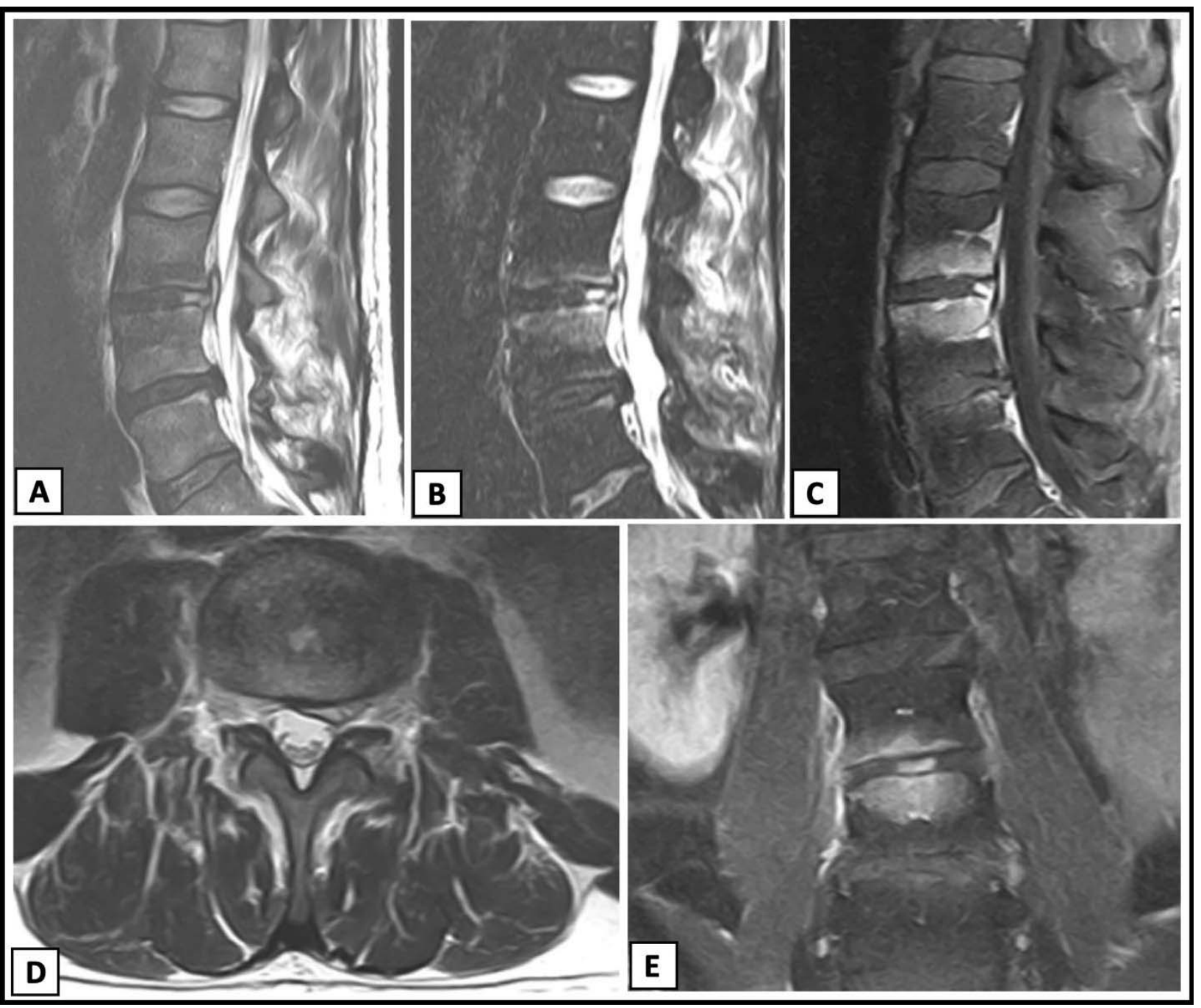

Figure 3. Case 2. Imaging 4 weeks postprocedure. (A) T2-weighted lumbar magnetic resonance imaging (MRI) without contrast, sagittal view demonstrating increased signal at L3-L4 with concern for epidural abscess. (B) Sagittal short tau inversion recovery (STIR) image demonstrating increased signal at L3-L4 with endplate changes. (C) T1-weighted lumbar MRI postcontrast, sagittal view demonstrating enhancement at the endplates of L3 and L4 with increased signal in the L3L4 disc. (D) T2-weighted lumbar MRI without contrast, axial view of L3-L4 demonstrating left paracentral disc material versus abscess. (E) T1-weighted lumbar MRI postcontrast, coronal view demonstrating endplate changes in vertebral bodies and disc enhancement at L3-L4.

tion using a Marrow Cellutions Bone Aspiration System (Ranfac Corporation). Sixty milliliters of bone marrow was aspirated and concentrated to 7 $\mathrm{mL}$ by using a bone marrow concentration device (EmCyte Corporation). Following administration of IV cefazolin, fluoroscopy was used for doubleneedle technique intradiscal injection of $2.5-3 \mathrm{~mL}$ of BMC at each level (Figure 4A-C).

Thirteen days following the procedure, he continued to have severe pain requiring opioid analgesia. His examination showed restricted and guarded range of motion in the lumbar spine with right-sided dural tension signs, but no focal neurological deficits. Early MRI of the lumbar spine at 2 weeks postprocedure showed mild increased T2 signal in the L4-5 high-intensity zone as well as an increase in the disc protrusion at L5-S1 (Figure 4D). Because of the development of fever and unremitting pain, he underwent a CT-guided biopsy that was positive for Cutibaceterium acnes (formerly Propionibacterium acnes) at L5-S1. His repeat MRI at 4 weeks postprocedure demonstrated increased endplate changes and central bone remodeling at L5-S1 (Figure 4E,F). At their peak, CRP and ESR were $82.9 \mathrm{mg} / \mathrm{L}$ and $39(\mathrm{~mm} / \mathrm{hr})$, respectively, while the white blood cell count remained within a normal range at $7.310^{9 /} \mathrm{L}$. He was treated with IV ceftriaxone for 42 days followed by oral amoxicillin for an additional 42 days.

At 3 months postprocedure, his blood work normalized, and he had improvement in his pain back to his preinjection baseline status. He was 


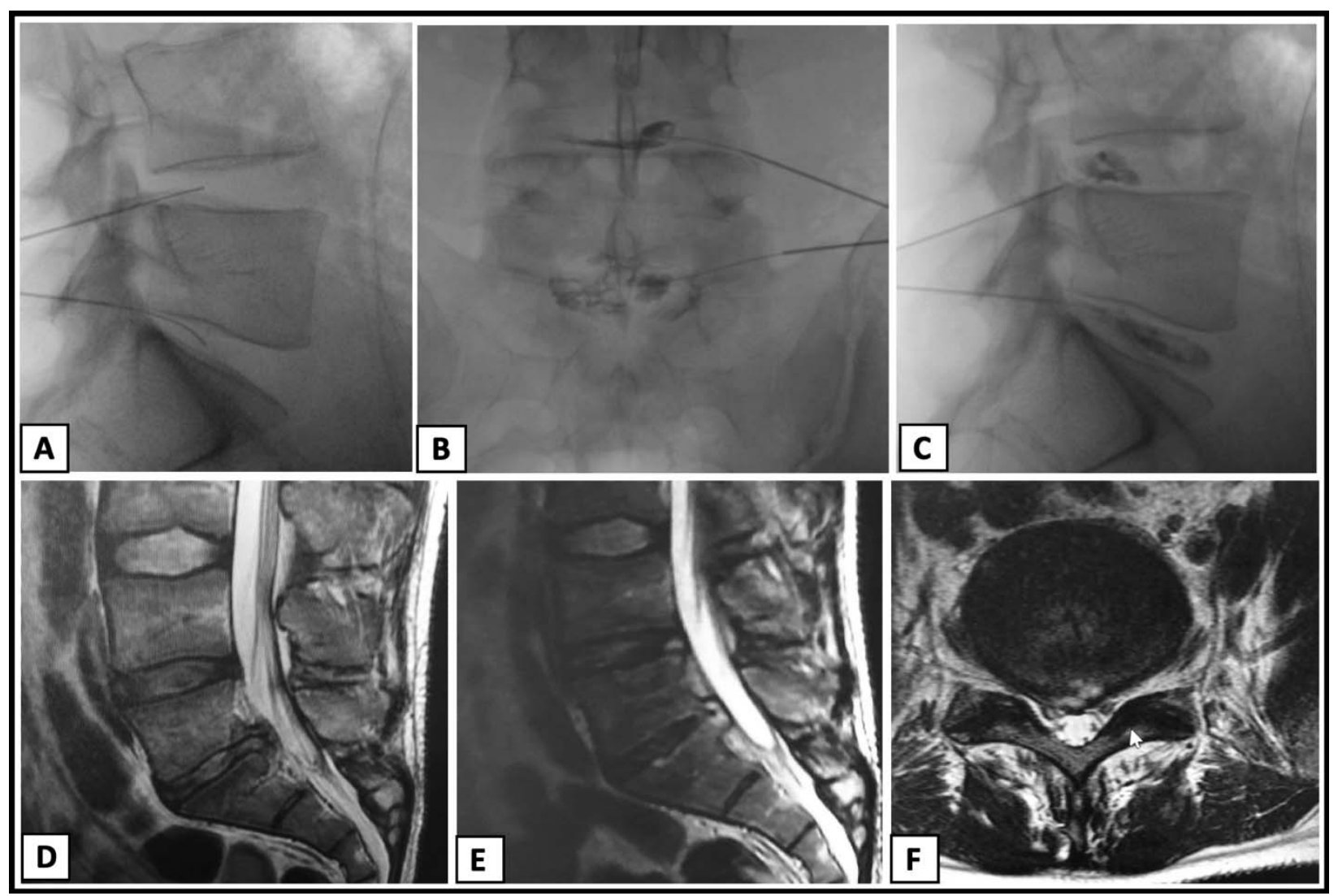

Figure 4. Case 3. (A) Lateral fluoroscopic image demonstrating L4-L5 and L5-S1 intradiscal needle placement. (B) Anteroposterior fluoroscopic view demonstrating L4-L5 and L5-S needle placement and discogram. (C) Lateral fluoroscopic view demonstrating L4-L5 and L5-S needle placement and discogram. (D) Two weeks postprocedure T2-weighted lumbar magnetic resonance imaging (MRI), sagittal view, demonstrating disc disease with minimal endplate edema at L5-S1. (E) Four weeks postprocedure T2-weighted lumbar MRI, sagittal view, demonstrating increased subchondral edema at the L5-S1 endplates and subtle central bone plate remodeling. (F) Four weeks postprocedure lumbar MRI, axial view, demonstrating large broad-based disc protrusion with associated annular fissure.

rarely using opioids and achieved pain control primarily with nonsteroidal anti-inflammatory drugs. He had no progressive neurological symptoms or continued fevers.

In summary, one case of confirmed discitis with $C$ acnes was identified, and two cases of suspected spondylodiscitis were identified. All cases used BMC as the injectate, and in all cases, one level of two was found to have a complication. A summary of characteristics can be found in Table 2 .

\section{DISCUSSION}

Our clinical experience and review of the published literature has demonstrated a small but notable risk of spondylodiscitis following intradiscal orthobiologic injections of PRP and/or BMC for lumbar disc disease. Here, we present three previously unpublished cases of complications following intradiscal injection with BMC. Review of the published literature identified two previous cases of spondylodiscitis following orthobiologic intra- discal injections, one following combination BMC and adipose injection and the second following leukocyte-poor PRP injections. From the reports that do exist in combination with the above series, it is possible that bone marrow-based injections may be more commonly associated with risk of infection and/or complication. However, more comprehensive multicenter retrospective data are needed to confirm this trend. Reasons for this potential increased risk are not entirely understood, but could be related to several factors. First, increased handling of the injectate may allow for possible contamination. In order to process bone marrow, it is transferred from the sterile field to a processing device and centrifuge. Although the systems used in the cases reported were closed, it is possible that these additional steps allow for potential contamination. Bacteria have been cultured from surgical devices left on sterile trays for greater than 30 minutes and with suspected inoculated circulating air. $^{45}$ It is possible that a similar occurrence is responsible for bacterial contamination during some 
Table 2. Review of case series patient characteristics.

\begin{tabular}{|c|c|c|c|}
\hline \multirow[b]{2}{*}{ Parameter } & \multicolumn{3}{|c|}{ Patient } \\
\hline & 1 & 2 & 3 \\
\hline Sex & M & M & M \\
\hline Age, y & 55 & 35 & 34 \\
\hline Prior surgery & No & $\begin{array}{l}\text { L4-5 microdiscectomy and } \\
\text { laminectomy in 2015; revision } \\
\text { in } 2016 \text { with postsurgical CSF } \\
\text { leak }\end{array}$ & No \\
\hline Prior steroids & $\begin{array}{l}\text { Methylprednisolone dose pack } 3 \\
\text { mo prior }\end{array}$ & $\begin{array}{l}\text { Corticosteroid epidural, date } \\
\text { unknown }\end{array}$ & Yes \\
\hline Previous injection & Lumbar facets with Regenokine & $\begin{array}{l}\text { Corticosteroid epidural, date } \\
\text { unknown }\end{array}$ & Yes, intradiscal LP-PRP twice \\
\hline Injectate & $\mathrm{BMC}$ & $\mathrm{BMC}$ & $\mathrm{BMC}$ \\
\hline IV antibiotics & Yes, cefazolin & Yes, cefazolin & Yes, cefazolin \\
\hline Antibiotics in contrast & Yes, $500 \mathrm{mcg}$ gentamycin & No & No \\
\hline Levels injected & $2, \mathrm{~L} 4-5, \mathrm{~L} 5-\mathrm{S} 1$ & 2, L3-5, L4-5 & 2, L4-5, L5-S1 \\
\hline Levels with complication & 1, L5-S1 & $1, \mathrm{~L} 3-4$ & $1, \mathrm{~L} 4-5$ \\
\hline Confirmed pathogen & No & $\begin{array}{l}\text { No, culture of fluid collection } \\
\text { negative }\end{array}$ & Yes, $C$ acnes \\
\hline $\operatorname{ESR}(\mathrm{mm} / \mathrm{hr})$ & 115.5 & 49 & 41 \\
\hline CRP $(\mathrm{mg} / \mathrm{L})$ & 35 & 4.9 & 82.9 \\
\hline WBC $\left(10^{9 /} \mathrm{L}\right)$ & 8.6 & 6.3 & 7.3 \\
\hline Fever & No & Yes & Yes \\
\hline MRI findings & Endplate edema & Endplate edema & Endplate edema \\
\hline Treatment & $\begin{array}{l}\text { IV cloxacillin and oral rifampicin } \\
\text { and moxifloxacin }\end{array}$ & $\begin{array}{l}\text { IV cefepime, ceftriaxone, and } \\
\text { steroids }\end{array}$ & $\begin{array}{l}\text { IV ceftriaxone and oral } \\
\text { amoxicillin }\end{array}$ \\
\hline Outcome & Clinical improvement & $\begin{array}{l}\text { Clinical improvement in pain after } \\
\text { systemic steroids }\end{array}$ & $\begin{array}{l}\text { Persistent pain leading to } \\
\text { consideration of surgical fusion }\end{array}$ \\
\hline
\end{tabular}

Abbreviations: BMC, bone marrow concentrate; CRP, C-reactive protein; CSF, cerebrospinal fluid; ESR, erythrocyte sedimentation rate; IV, intravenous; LP, leukocyte poor; MRI, magnetic resonance imaging; PRP, platelet-rich plasma; WBC, white blood cell count.

portion of the BMC processing. In addition, rather than a peripheral blood draw using up to an 18 gauge or smaller-sized needle, bone marrow aspirations are typically performed with larger, 11- or 15gauge bone marrow biopsy needles. Theoretically, the larger size and surface area may be a higher risk for contamination despite sterile technique.

Another possible explanation is new data regarding the microbiota within various environments in the body. One of the most compelling theories relates to recent research identifying an intradiscal microbiome. The assumption that the intradiscal space is a sterile environment has recently come into question. $^{46,47}$ There appears to exist a complex microbiome within the IVD with differing distributions and diversity of bacterial populations in healthy discs versus in discs with degeneration and herniation. This suggests a possible dysbiosis theory underlying chronic disc degeneration. ${ }^{46,48}$ It is possible that the combination of growth factors causes an imbalance and overgrowth of the innate microbiome already present within the disc. Reported cases of spontaneous discitis ${ }^{49,50}$ further support a possible skin/gut/spine microbiome axis and dysbiosis model. ${ }^{48}$ This raises additional questions regarding other microbiomes within the body. It is possible that the bone marrow space within the pelvis also harbors its own unique microbiome, thereby allowing the transfer of dormant bacteria into the discs during BMC injection. Although, to our knowledge, a discrete microbiome of the bone marrow space has not been identified, studies do indicate a gut microbiome influence on aging of circulating neutrophils ${ }^{47}$ and the prevention of graft-versus-host disease following allogeneic bone marrow transplantation in patients with gut microbiome suppression, ${ }^{51}$ indicating some degree of a gut/bone marrow axis.

Another consideration is that one of the presented cases did not have a confirmed microorganism from his intradiscal, CT-guided biopsy and culture. The patient did not significantly improve clinically with IV antibiotics; however, they made a marked recovery following administration of high-dose corticosteroids. This raises the possibility of an extreme inflammatory reaction to either the cells within the BMC or an additive within the injectate. It is also possible the aspiration was not adequate for culture; however, it emphasizes the importance of understanding the different growth factors and 
cell types within biologic injectates as well as their specific effects within various environments in vivo. While there is great enthusiasm for orthobiologic treatment options, this enthusiasm has to be tempered with ongoing safety and clinical outcome studies to weigh the risk/reward for patients.

There are several key clinical takeaways from the available data. First, patients in whom infection was identified early went on to do reasonably well clinically and did not need surgical fusion. Crescendo pain should signal the clinician to evaluate for infection early in the postprocedure time frame. For many patients, intradiscal biologic injection leads to an immediate increase in postprocedure pain; however, this typically resolves by day seven. ${ }^{12,22,23}$ Patients presenting with infection often have an improvement in pain followed by crescendo pain within 1 to 2 weeks, many times with continued fluctuation and no overall improved trajectory. In addition, MRI imaging features pathognomonic for infection often lag behind the clinical presentation of acute infection. ${ }^{52}$ Therefore, imaging alone is insufficient for diagnosis, and any patient with persistent pain 2 weeks postinjection requiring continued use of opioid analgesics should be evaluated for suspected infection. Patients may not always present with fever, and leukocyte counts can be within the normal range. However, ESR and CRP are useful markers, and elevated levels are highly predictive of spondylodiscitis, especially when co-occurring with fever and changes on MRI. ${ }^{53}$ When seen, concerning changes on MRI include increased endplate signal changes in $\mathrm{T} 2$ weighing and loss of endplate definition in T1-weighted sequences. ${ }^{54}$ If there is concern for infection, it is recommended that the patient undergo a CT-guided biopsy before initiating antibiotics. This should be followed by coverage with a broad-spectrum antibiotic, such as ceftriaxone, while awaiting results. In addition, because overgrowth of $C$ acnes may be the causative agent in many of these cases, cultures need to be performed for at least 14 days under both aerobic and anaerobic conditions so it is not missed. ${ }^{55}$

Regarding mitigation strategies, among others, $C$ acnes discitis has been previously reported following orthobiologic injection, ${ }^{26}$ was present in the CTguided biopsy of one case presented above, and has been identified as the causative pathogen in cases of spontaneous discitis. ${ }^{50}$ Although the introduction of bacteria through needle contamination is theoretically possible, all procedures were performed with a two-needle technique and strict sterile technique. This technique has been shown to decrease the rate of infection following discography from $2.7 \%$ to $0.7 \%{ }^{56}$ and therefore should be used during biologic intradiscal injection. The addition of antibiotics effective against $C$ acnes to contrast dye may further reduce the risk for intradiscal infection and should be studied to determine dosing as well as potential toxicity to the biologic injectate. Although evidence is not sufficient to recommend the addition of antibiotics to contrast agents when using a twoneedle sterile technique for discography alone, ${ }^{57}$ gentamicin has demonstrated preserved biological activity when added to iohexol in vitro ${ }^{58}$ and therefore may be of value in the setting of biologic intradiscal injection.

A recent study evaluating in vitro growth of $C$ acnes in response to the application of cefazolin and various PRP preparations demonstrated an incomplete suppression of $C$ acnes with this antibiotic. Although cefazolin is a suboptimal antibiotic choice, there was a significant reduction in bacterial load when exposed to PRP with a higher neutrophil content. ${ }^{59}$ Therefore, it would appear that the antimicrobial properties in a leukocyte-rich PRP preparation may have the potential to suppress intradiscal infection and/or dysbiosis. As this is the only intradiscal orthobiologic with both doubleblind randomized controlled trial and long-term outcomes data, ${ }^{11}$ it is currently the preferred injectate for use in the intradiscal space in our practice.

Our review does have some limitations. The reported infectious complications are from single case reports and do not reflect the number of uncomplicated procedures performed by those clinicians and practices. This again highlights the importance for a larger, multi-institution review to identify a more accurate complication rate for these procedures. In addition, the majority of studies included in the review are case series with retrospective review. Some patients may have been lost to follow-up, thereby making it difficult to fully ascertain rates of complication. Our review also does not include studies of allogenic orthobiologic injections, which are emerging as promising treatments ${ }^{13,60}$ but may present additional complication risks, such as human leukocyte antigen 
mismatch or other considerations outside the scope of this paper.

\section{CONCLUSION}

A historically difficult pathology to treat, intradiscal injections of biologic substances show promise in treating discogenic back pain. As more knowledge is gathered about the complex microenvironments within the spine, including the microbiome, it is imperative to critically evaluate the in vivo effects of injectates with biological activity. Furthermore, although overall very safe within the scope of invasive procedures, cases of spondylodiscitis have been reported following intradiscal orthobiologic injections, and a multicenter survey is needed to more accurately quantify the true incidence of these complications. At present, basic science data support the use of a leukocyte-rich PRP for safety optimization when performing orthobiologic intradiscal injections. Further studies are needed through multicenter national registries to track not only clinical outcomes of these therapies but also complication rates and optimize injectate preparations. In the meantime, clinicians performing these procedures are encouraged to share their clinical experiences and report complications when they occur so that clinicians can make informed decisions to optimize patient safety.

\section{REFERENCES}

1. Taher F, Essig D, Lebl DR, et al. Lumbar degenerative disc disease: current and future concepts of diagnosis and management. Adv Orthop. 2012;2012:7. doi:10.1155/2012/ 970752

2. Hoy D, Brooks P, Blyth F, Buchbinder R. The epidemiology of low back pain. Best Prac Res Clin Rheumatol. 2010;24(6):769-781.

3. Verrills P, Nowesenitz G, Barnard A. Prevalence and characteristics of discogenic pain in tertiary practice: 223 consecutive cases utilizing lumbar discography. Pain Med. 2015;16(8):1490-1499.

4. Mwale F, Roughley P, Antoniou J. Distinction between the extracellular matrix of the nucleus pulposus and hyaline cartilage: a requisite for tissue engineering of intervertebral disc. Eur Cell Mater. 2004;8(58):63-64.

5. Stokes IA, Iatridis JC. Mechanical conditions that accelerate intervertebral disc degeneration: overload versus immobilization. Spine. 2004;29(23):2724. doi:10.1097/ 01.brs.0000146049.52152.da

6. Kepler CK, Ponnappan RK, Tannoury CA, Risbud MV, Anderson DG. The molecular basis of intervertebral disc degeneration. Spine J. 2013;13(3):318-330.

7. Fujii K, Yamazaki M, Kang JD, et al. Discogenic back pain: literature review of definition, diagnosis, and treatment. JBMR Plus. 2019;3(5):e10180-e10180. doi:10.1002/jbm4.10180

8. Smith JS, Sidhu G, Bode K, et al. Operative and nonoperative treatment approaches for lumbar degenerative disc disease have similar long-term clinical outcomes among patients with positive discography. World Neurosurg. 2014;82(5):872-878.

9. Wang SZ, Rui YF, Lu J, Wang C. Cell and molecular biology of intervertebral disc degeneration: current understanding and implications for potential therapeutic strategies. Cell Prolif. 2014;47(5):381-390.

10. Wei A, Shen B, Williams L, Diwan A. Mesenchymal stem cells: potential application in intervertebral disc regeneration. Transl Pediatr. 2014;3(2):71-90.

11. Tuakli-Wosornu YA, Terry A, Boachie-Adjei K, et al. Lumbar intradiskal platelet-rich plasma (PRP) injections: a prospective, double-blind, randomized controlled study. $P M R$. 2016;8(1):1-10; quiz 10.

12. Pettine KA, Suzuki RK, Sand TT, Murphy MB. Autologous bone marrow concentrate intradiscal injection for the treatment of degenerative disc disease with three-year follow-up. Int Orthop. 2017;41(10):2097-2103.

13. Noriega DC, Ardura F, Hernández-Ramajo R, et al. Intervertebral disc repair by allogeneic mesenchymal bone marrow cells: a randomized controlled trial. Transplantation. 2017;101(8):1945-1951.

14. Orozco L, Soler R, Morera C, Alberca M, Sánchez A, García-Sancho J. Intervertebral disc repair by autologous mesenchymal bone marrow cells: a pilot study. Transplantation. 2011;92(7):822-828.

15. Elabd C, Centeno CJ, Schultz JR, Lutz G, Ichim T, Silva FJ. Intra-discal injection of autologous, hypoxic cultured bone marrow-derived mesenchymal stem cells in five patients with chronic lower back pain: a long-term safety and feasibility study. J Transl Med. 2016;14:253. doi:10.1186/s12967-0161015-5

16. Centeno C, Markle J, Dodson E, et al. Treatment of lumbar degenerative disc disease-associated radicular pain with culture-expanded autologous mesenchymal stem cells: a pilot study on safety and efficacy. J Transl Med. 2017;15(1):197. doi:10.1186/s12967-017-1300-y

17. Navani A, Manchikanti L, Albers SL, et al. Responsible, safe, and effective use of biologics in the management of low back pain: American Society of Interventional Pain Physicians (ASIPP) guidelines. Pain Physician. 2019;22(1s):S1-S74.

18. Manchikanti L, Centeno CJ, Atluri S, et al. Bone marrow concentrate (BMC) therapy in musculoskeletal disorders: evidence-based policy position statement of American Society of Interventional Pain Physicians (ASIPP). Pain Physician. 2020;23(2):E85-E131.

19. Comella K, Blas JAP, Ichim T, Lopez J, Limon J, Moreno RC. Autologous stromal vascular fraction in the intravenous treatment of end-stage chronic obstructive pulmonary disease: a phase I trial of safety and tolerability. J Clin Med Res. 2017;9(8):701-708.

20. Haufe SM, Mork AR. Intradiscal injection of hematopoietic stem cells in an attempt to rejuvenate the intervertebral discs. Stem Cells Dev. 2006;15(1):136-137.

21. Kumar H, Ha DH, Lee EJ, et al. Safety and tolerability of intradiscal implantation of combined autologous adiposederived mesenchymal stem cells and hyaluronic acid in patients with chronic discogenic low back pain: 1-year follow-up of a 
phase I study. Stem Cell Res. Ther. 2017;8(1):262. doi:10.1186/ s13287-017-0710-3

22. Pettine K, Suzuki R, Sand T, Murphy M. Treatment of discogenic back pain with autologous bone marrow concentrate injection with minimum two year follow-up. Int Orthop. 2016;40(1):135-140.

23. Pettine KA, Murphy MB, Suzuki RK, Sand TT. Percutaneous injection of autologous bone marrow concentrate cells significantly reduces lumbar discogenic pain through 12 months. Stem Cells. 2015;33(1):146-156.

24. Subach BR, Copay AG, Martin MM, Schuler TC, DeWolfe DS. Epidural abscess and cauda equina syndrome after percutaneous intradiscal therapy in degenerative lumbar disc disease. Spine J. 2012;12(11):e1-4.

25. Wolff M, Shillington JM, Rathbone C, Piasecki SK, Barnes B. Injections of concentrated bone marrow aspirate as treatment for discogenic pain: a retrospective analysis. $B M C$ Musculoskelet Disord. 2020;21(1):135. doi:10.1186/s12891-0203126-7

26. Beatty NR, Lutz C, Boachie-Adjei K, Leynes TA, Lutz C, Lutz G. Spondylodiscitis due to Cutibacterium acnes following lumbosacral intradiscal biologic therapy: a case report. Regen Med. 2019;14(9):823-829.

27. Lutz GE. Increased nuclear T2 signal intensity and improved function and pain in a patient one year after an intradiscal platelet-rich plasma injection. Pain Med. 2017;18(6):1197-1199.

28. Levi D, Horn S, Tyszko S, Levin J, Hecht-Leavitt C, Walko E. Intradiscal platelet-rich plasma injection for chronic discogenic low back pain: preliminary results from a prospective trial. Pain Med. 2016;17(6):1010-1022.

29. Kirchner F, Anitua E. Intradiscal and intra-articular facet infiltrations with plasma rich in growth factors reduce pain in patients with chronic low back pain. J Craniovertebr Junction Spine. 2016;7(4):250-256.

30. Akeda K, Ohishi K, Masuda K, et al. Intradiscal injection of autologous platelet-rich plasma releasate to treat discogenic low back pain: a preliminary clinical trial. Asian Spine J. 2017;11(3):380-389.

31. Navani A, Hames A. Platelet-rich plasma injections for lumbar discogenic pain: a preliminary assessment of structural and functional changes. Techniques in Regional Anesthesia and Pain Management. 2015;19(1):38-44.

32. Cheng J, Santiago KA, Nguyen JT, Solomon JL, Lutz GE. Treatment of symptomatic degenerative intervertebral discs with autologous platelet-rich plasma: follow-up at 5-9 years. Regen Med. 2019;14(9):831-840.

33. Jain D, Goyal T, Verma N, Paswan AK, Dubey RK. Intradiscal platelet-rich plasma injection for discogenic low back pain and correlation with platelet concentration: a prospective clinical trial. Pain Med. 2020;21(11):2719-2725.

34. Basso M, Cavagnaro L, Zanirato A, et al. What is the clinical evidence on regenerative medicine in intervertebral disc degeneration? Musculoskelet Surg. 2017;101(2):93-104.

35. Bhangare KP, Kaye AD, Knezevic NN, Candido KD, Urman RD. An analysis of new approaches and drug formulations for treatment of chronic low back pain. Anesthesiol Clin. 2017;35(2):341-350.

36. Charneux L, Demoulin C, Vanderthomment M, et al. Platelet-rich plasma (PRP) and disc lesions: a review of the literature. Neurochirurgie. 2017;63(6):473-477.

37. Gou S, Oxentenko SC, Eldrige JS, et al. Stem cell therapy for intervertebral disk regeneration. Am J Phys Med Rehabil. 2014;93(11 Suppl 3):S122-S131.

38. Law L, Hunt CL, van Wijnen AJ, et al. Office-based mesenchymal stem cell therapy for the treatment of musculoskeletal disease: a systematic review of recent human studies. Pain Med. 2019;20(8):1570-1583.

39. Migliorini F, Rath B, Tingart M, Baroncini A, Quack V, Eschweiler J. Autogenic mesenchymal stem cells for intervertebral disc regeneration. Int Orthop. 2019;43(4):1027-1036.

40. Monfett M, Harrison J, Boachie-Adjei K, Lutz G. Intradiscal platelet-rich plasma (PRP) injections for discogenic low back pain: an update. Int Orthop. 2016;40(6):1321-1328.

41. Sakai D, Andersson GB. Stem cell therapy for intervertebral disc regeneration: obstacles and solutions. Nat Rev Rheumatol. 2015;11(4):243-256.

42. Urits I, Viswanath O, Galasso AC, et al. Platelet-rich plasma for the treatment of low back pain: a comprehensive review. Curr Pain Headache Rep. 2019;23(7):52. doi:10.1007/ s11916-019-0797-6

43. Zhao L, Manchikanti L, Kaye AD, Abd-Elsayed A. Treatment of discogenic low back pain: current treatment strategies and future options-a literature review. Curr Pain Headache Rep. 2019;23(11):86. doi:10.1007/s11916-019-0821-x

44. Akeda K, Yamada J, Linn ET, Sudo A, Masuda K. Platelet-rich plasma in the management of chronic low back pain: a critical review. J Pain Res. 2019;12:753-767. doi:10. 2147/JPR.S153085

45. Menekse G, Kuscu F, Suntur BM, et al. Evaluation of the time-dependent contamination of spinal implants: prospective randomized trial. Spine (Phila Pa 1976). 2015;40(16):12471251.

46. Rollason J, McDowell A, Albert HB, et al. Genotypic and antimicrobial characterisation of Propionibacterium acnes isolates from surgically excised lumbar disc herniations. BioMed Res Int. 2013;2013:530382. doi:10.1155/2013/530382

47. Zhang D, Chen G, Manwani D, et al. Neutrophil ageing is regulated by the microbiome. Nature. 2015;525(7570):528532.

48. Rajasekaran S, Soundararajan DCR, Tangavel C, et al. Human intervertebral discs harbour a unique microbiome and dysbiosis determines health and disease. Eur Spine J. 2020;29(7):1621-1640.

49. Friedman JA, Maher CO, Quast LM, McClelland RL, Ebersold MJ. Spontaneous disc space infections in adults. Surg Neurol. 2002;57(2):81-86.

50. Honan M, White GW, Eisenberg GM. Spontaneous infectious discitis in adults. Am J Med. 1996;100(1):85-89.

51. Vossen JM, Guiot HF, Lankester AC, et al. Complete suppression of the gut microbiome prevents acute graft-versushost disease following allogeneic bone marrow transplantation. PloS One. 2014;9(9):e105706. doi:10.1371/journal.pone.0105706

52. Schindler O, Wilson-MacDonald J. Acute MRI changes in infectious discitis: report on two cases. Eur Spine $J$. 1995;4(6):360-361.

53. Kihira S, Koo C, Mahmoudi K, et al. Combination of imaging features and clinical biomarkers predicts positive pathology and microbiology findings suggestive of spondylodiscitis in patients undergoing image-guided percutaneous biopsy. Am J Neuroradiol. 2020;41(7):1316-1322.

54. Modic MT, Feiglin DH, Piraino DW, et al. Vertebral osteomyelitis: assessment using MR. Radiology. 1985;157(1):157-166. 
55. Rentenaar RJ, Kusen SM, Riemens-van Zetten G, van Mourik MS. Detection of clinical Cutibacterium acnes isolates in different Becton Dickinson blood culture vials. $J$ Clin Microbiol. 2018;56(1). doi:10.1128/JCM.01486-17

56. Fraser RD, Osti OL, Vernon-Roberts B. Discitis after discography. J Bone Joint Surg Br. 1987;69(1):26-35.

57. Sharma SK, Jones JO, Zeballos PP, Irwin SA, Martin TW. The prevention of discitis during discography. Spine $J$. 2009;9(11):936-943.

58. Klessig HT, Showsh SA, Sekorski A. The use of intradiscal antibiotics for discography: an in vitro study of gentamicin, cefazolin, and clindamycin. Spine. 2003;28(15):1735-1738.

59. Prysak MH, Lutz CG, Zukofsky TA, Katz JM, Everts PA, Lutz GE. Optimizing the safety of intradiscal platelet-rich plasma: an in vitro study with Cutibacterium acnes. Regen Med. 2019;14(10):955-967.

60. Amirdelfan K, Bae H, McJunkin T, et al. Allogeneic mesenchymal precursor cells treatment for chronic low back pain associated with degenerative disc disease: a prospective randomized, placebo-controlled 36-month study of safety and efficacy. Spine J. 2021;21(2):212-230.

Disclosures and COI: No authors have any relevant disclosures.

Corresponding Author: Mairin Jerome, MD, Regenerative SportsCare Institute, 62 East 88th Street, Ground Floor, New York, NY 10128. Phone: (646) 870-7997; Fax: (646) 870-7990; Email: jeromem@regensportscare.com.

Published 30 April 2021

This manuscript is generously published free of charge by ISASS, the International Society for the Advancement of Spine Surgery. Copyright (c) 2021 ISASS. To see more or order reprints or permissions, see http://ijssurgery.com. 